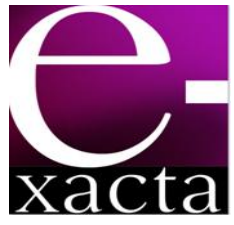

ISSN: 1984-3151

\section{TRATAMENTO DE RESíDUOS DO MÉTODO DE MOHR UTILIZANDO EVAPORADOR SOLAR E REDUÇÃO COM BISSULFITO}

\author{
TREATMENT OF WASTE OF MOHR METHOD USING SOLAR \\ EVAPORATOR AND REDUCTION WITH BISULFITE
}

\author{
Juliana Monteiro da Silva'; Paulo Henrique Medeiros Theophilo²; Geovany Rocha \\ Torres $^{3}$; Simone da Silveira Sá Borges ${ }^{4}$
}

1 Mestranda em Química. Universidade Federal do Ceará. Fortaleza, CE. juliana monteiro @hotmail.com.

2 Mestre em Química. Universidade Federal do Ceará, 2014. Técnico em Química na Universidade Federal do Ceará. Fortaleza, Ceará. ppaulohenrique@hotmail.com.

3 Mestre em Desenvolvimento e Meio Ambiente. Universidade Federal do Ceará, 2008. Técnico de Laboratório da Universidade Federal do Ceará. Fortaleza, Ceará. geo@ufc.br.

4 Doutora em Físico-química. Universidade de São Carlos, 1995. Professora Associado I na Universidade Federal do Ceará. Fortaleza, Ceará. simone@ufc.br.

Recebido em: 08/09/2015 - Aprovado em: 20/11/2015 - Disponibilizado em: 30/11/2015

RESUMO: O quadro econômico atual estimula o consumismo e traz consigo uma alta produção de resíduos, estes muitas vezes ignorados até que acarretem ameaças e conflitos ambientais mais graves às comunidades. As universidades não estão isentas dessa problemática, pois nela há diversos locais de produção e acúmulo de resíduos. O correto gerenciamento destes resíduos é essencial para minimizar os riscos ao meio ambiente, bem como à comunidade acadêmica. Atualmente, o maior desafio é com os resíduos laboratoriais, pois são de uma enorme complexidade e diversidade e o tratamento desses resíduos, muitas vezes envolve a geração de um novo material residual. Assim, este trabalho tem como objetivo o estudo da pré-concentração do resíduo do Método de Mohr, para posterior tratamento, em um evaporador solar construído especificamente para tal fim, tornando o gerenciamento do referido resíduo mais econômico, limpo e seguro. Com a utilização do evaporador solar houve a minimização de riscos de acidentes, bem como a economia com energia elétrica em $R \$ 862,87$. O evaporador solar se mostrou muito eficiente, pois a água resultante da evaporação, devido a baixa concentração de cromo total remanescente $\left(0,235 \mathrm{mgL}^{-1}\right)$ e $\mathrm{pH}$, pode ser reutilizada para manutenções de jardins reduzindo a utilização de água tratada para este fim.

PALAVRAS-CHAVE: Resíduo. Evaporador solar. Economia.

ABSTRACT: The current economic framework encourages consumerism and brings with it a high production of waste, these often ignored until they cause in most serious environmental conflicts and threats to communities. Universities are not exempt from this problem, because in it there are several places of production and accumulation of waste. The correct management of these wastes is essential to minimize the risk to the environment, as well as the academic community. Currently, the greatest challenge is with the laboratory waste, because they are of enormous complexity and diversity and treatment of such waste, often involves the generation of a new residual material. This work aims the study of pre-concentration of waste of Mohr method, for subsequent treatment, in a solar evaporator built specifically for this purpose, making the management of this waste more economical, clean and safe. With the use of solar evaporator was minimizing the risk of accidents and the economy with electricity at $R \$ 862,87$. The solar evaporator proved to be very efficient, because water resulting from evaporation, due to low concentration of total chromium remnant $\left(0.235 \mathrm{mgL}^{-1}\right)$ and $\mathrm{pH} 7$, can be reused for garden maintenance reducin the use of treated water for this purpose.

KEYWORDS: Waste. Solar evaporator. Economy. 


\section{INTRODUÇÃO}

O quadro econômico atual que estimula 0 desenvolvimento de novos produtos e o consumismo traz consigo uma alta produção de resíduos, e estes muitas vezes são ignorados até que acarretem ameaças, iniquilidades e conflitos ambientais mais graves às comunidades (PENATTI, GUIMARÃES, SILVA, 2008). As universidades não estão isentas desta problemática, pois nela há diversos locais de produção e acúmulo de resíduos, como por exemplo, laboratórios de ensino, pesquisa e extensão e estes são muitas vezes armazenados no próprio laboratório, quando não os descartam de maneira incorreta na pia ou lixo.

Como as universidades são instituições responsáveis pela formação de seus estudantes e, consequentemente, influenciam seu comportamento como cidadão, estas devem se conscientizar a respeito da geração, armazenamento, tratamento e disposição final de seus resíduos. Então, em 1990, as universidades começaram a discutir o gerenciamento de resíduos laboratoriais e desde essa época vem realizando trabalhos que tem como objetivos gerenciar e tratar seus materiais residuais de forma a diminuir o impacto causado ao meio ambiente (GIOLI-LIMA, LIMA, 2003).

Dessa forma, foi criado em 2005 na Universidade Federal do Ceará (UFC) o Programa Gerenciamento de Resíduos (PROGERE), que reúne um conjunto de procedimentos e ações visando a implantação de um sistema integrado de coleta seletiva, redução, reutilização, reciclagem e destinação final dos diversos tipos de resíduos gerados nas atividades de ensino, pesquisa, extensão e administração da UFC. O PROGERE foi estruturado em três plataformas de acordo com o desenvolvimento das ações específicas: gerenciamento de resíduos recicláveis, gerenciamento de resíduos laboratoriais e educação socioambiental.
O gerenciamento de resíduos laboratoriais é o maior desafio para o Programa, pois estes são de uma enorme complexidade e diversidade; e o tratamento desses resíduos, muitas vezes envolve a geração de um novo material residual. Assim, o PROGERE tem buscado criar e/ou aprimorar metodologias de tratamento para os principais resíduos gerados nos laboratórios de ensino, pesquisa e extensão da UFC.

Neste trabalho será abordado o resíduo oriundo do método de Mohr, que é utilizado nas disciplinas experimentais de Química Analítica Quantitativa para os cursos dos diversos Centros e Faculdades da UFC. O método de Mohr é um método analítico clássico argentimétrico de volumetria de precipitação direta, aplicado para quantificar íons cloreto, brometo e iodeto em uma amostra, sendo mais comumente aplicado ao íon cloreto.

Esse método tem grande importância, pois a determinação do íon cloreto é utilizada no controle de qualidade em diversos setores como, por exemplo, controle de qualidade de água para consumo humano, indústria farmacêutica, indústria de alimentos, controle de estações de tratamento de esgoto, entre outros. O resíduo originário desse método é formado por um sobrenadante, objeto de estudo deste trabalho, contendo, principalmente, o íon cromato $\left(\mathrm{CrO}_{4}{ }^{2-}\right)$, que contém íon cromo com estado oxidação $6+$, e um precipitado constituído por cloreto de prata $(\mathrm{AgCl})$ e cromato de prata $\left(\mathrm{Ag}_{2} \mathrm{CrO}_{4}\right)$.

O $\mathrm{Cr}(\mathrm{VI})$ penetra com facilidade por meio da membrana celular, e é convertido em $\mathrm{Cr}$ (III). Por sua vez, o $\mathrm{Cr}$ (III) é solúvel somente em baixos valores de $\mathrm{pH}$, e estes são geralmente menores do que aqueles normalmente encontrados em sistemas biológicos, ou quando complexado com moléculas orgânicas de baixo peso molecular, que possuem pouca mobilidade através da membrana celular (GUTTERRE, SILVA, DETTIMER, 2011). A conversão do $\mathrm{Cr}(\mathrm{VI})$ a $\mathrm{Cr}$ (III) no organismo pode acarretar em danos à estrutura 
celular e também causar um desequilíbrio devido ao aumento da concentração de $\operatorname{Cr}($ III), que passa a ser tóxico em níveis mais elevados (PAULINHO, 1993). O cromo também se apresenta tóxico a vida aquática, dependendo das espécies, tempo de exposição e fatores ambientais como temperatura, $\mathrm{pH}$, oxigênio dissolvido e dureza.

Há cinco anos o resíduo do método de Mohr produzido no Departamento de Química Analítica e Físico-química (DQAFQ) da UFC não tinha destino adequado. A partir de então foi implementada a coleta desse material residual no DQAFQ, sendo armazenado para futuro tratamento e descarte ambientalmente correto. Paralelamente a isso, foi realizado um trabalho de conscientização dos corpos discente, docente e técnicos de laboratório que estariam envolvidos na geração desse resíduo (OLIVEIRA, et al., 2010).

Foi realizada também a estimativa da quantidade média desse resíduo gerado no Laboratório de Química Analítica Quantitativa constatando-se que são produzidos $8 \mathrm{~L}$ por semestre (Cavalcante, Silva, Borges, 2012), que originou até 2014.2 um estoque de $116 \mathrm{~L}$ do resíduo.

O objetivo deste trabalho é o estudo da préconcentração do resíduo do Método de Mohr, para posterior tratamento, em um evaporador solar construído especificamente para tal fim, tornando o gerenciamento do referido resíduo mais econômico, limpo e seguro.

\section{MATERIAIS E MÉTODOS}

\subsection{Coleta e armaZenamento dos Resíduos}

Para a coleta dos resíduos foram utilizados frascos âmbar de reagentes com capacidade para um litro (FIG. 1). Esses frascos foram submetidos à tríplice lavagem bem como retirada do rótulo. Para a correta identificação dos resíduos, foi elaborada uma etiqueta exclusiva (FIG. 2), onde são colocados os seguintes dados: ponto gerador, nome do resíduo e seus constituintes principais, data que 0 frasco foi completado e o responsável pela coleta. Os resíduos foram armazenados em local específico de acesso restrito.

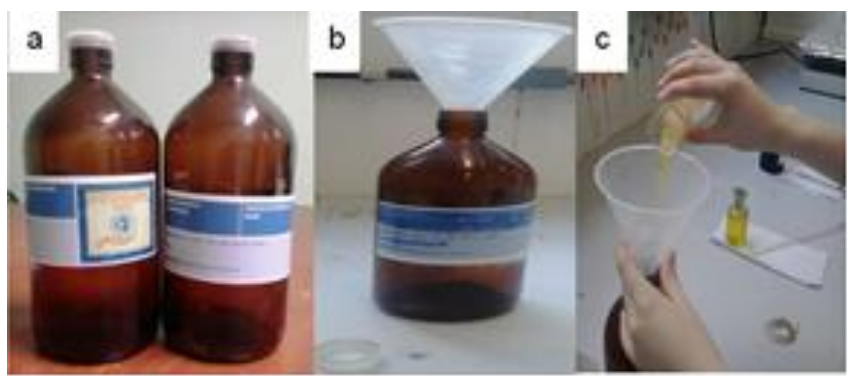

Figura 1 - (a) Frascos para coleta, (b) Frasco pronto para coleta, (c) Coleta do resíduo.

Fonte: Elaborado pelos autores

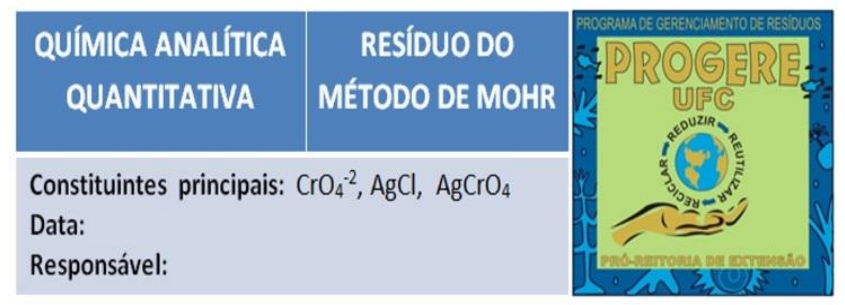

Figura 2 - Etiqueta para identificação do resíduo.

Fonte: Elaborado pelos autores

\subsection{TRATAMENTO PRELIMINAR DO RESíDUO}

O material residual do método de Mohr é constituído de uma parte sólida (precipitado) e uma parte líquida (sobrenadante). A parte sólida é composta por dois precipitados, o $\mathrm{AgCl}$ e $\circ \mathrm{Ag}_{2} \mathrm{CrO}_{4}$, e o sobrenadante por $\mathrm{CrO}_{4}{ }^{2-}$, com cromo no estado de oxidação $6+$.

Inicialmente, o sobrenadante é separado do precipitado por decantação e em seguida com o auxilio de um papel de filtro qualitativo, o resíduo é filtrado. Então, o precipitado é levado para secar na 
estufa e depois armazenado para futuro tratamento. $\mathrm{O}$ filtrado, contendo $\mathrm{Cr}(\mathrm{VI})$, é armazenado em novos frascos para, então, ser encaminhado à préconcentração.

\subsection{PrÉ-CONCENTRAÇÃo}

Normalmente a pré-concentração de um resíduo aquoso é feita em chapa aquecedora, entretanto este processo é dispendioso, demandando gastos com energia elétrica (chapa aquecedora e exaustor), controle de temperatura e velocidade de agitação e ainda o elevado risco de acidentes devido à alta temperatura de operação, principalmente quando se trabalha com um grande volume de resíduos.

Dessa forma, foi construído um evaporador solar para que o volume dos resíduos fosse reduzido ao máximo antes do tratamento (pré-concentração) economizando energia elétrica, além de reduzir os riscos de acidentes.

O evaporador (FIG. 3) é feito de madeira, na forma de trapézio, tem $115 \mathrm{~cm}$ de comprimento, $50,1 \mathrm{~cm}$ de largura, $41,3 \mathrm{~cm}$ de altura na parte de trás e $22,7 \mathrm{~cm}$ de altura na parte da frente. A tampa é constituída de vidro e tem $115 \mathrm{~cm}$ de comprimento e $53 \mathrm{~cm}$ de largura. O interior do evaporador foi submetido à pintura na cor preta e completamente coberto por resina. Foram colocadas placas de metal na base para maior acúmulo de energia. Há um cano de policloreto de vinila (PVC), cortado ao meio longitudinalmente, que fica localizado na parte frontal para condensação da água, sendo esta recolhida em um recipiente fechado.

No evaporador localizado em um local protegido de intempéries, que recebe uma grande quantidade de luz solar, foram dispostos 5 béqueres para receber 0 filtrado. Os frascos com os filtrados foram transportados até o evaporador e o seu conteúdo cuidadosamente despejado nos béqueres, até que o volume total destes estivesse completo. Para maior segurança e melhor deslocamento, os frascos contendo os filtrados foram armazenados próximo ao local onde estava localizado o evaporador.

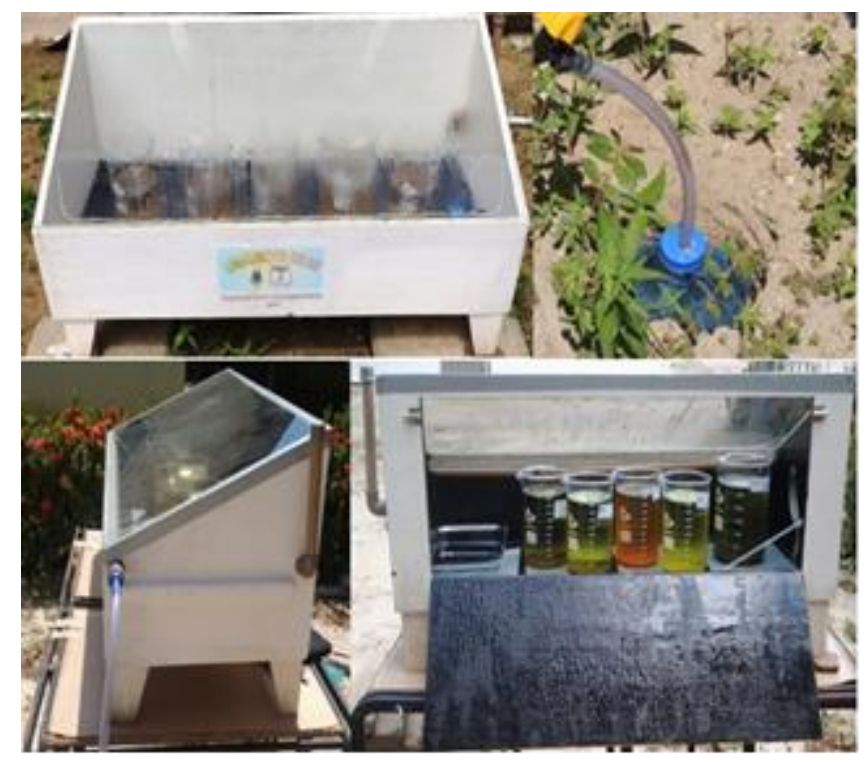

Figura 3 - Evaporador solar.

Fonte: Elaborado pelos autores

À medida que ocorria a evaporação era acrescentado mais filtrado até que o mesmo apresentasse uma coloração amarela escura e metade da capacidade do béquer, estando assim, apto para tratamento.

\subsection{TRATAMENTO do RESíduo}

O sobrenadante contendo $\mathrm{Cr}(\mathrm{VI})$ foi tratado seguindo os procedimentos de redução e precipitação apresentados em SILVA (2014).

Dispondo o filtrado pré-concentrado em um béquer, adiciona-se $\mathrm{NaHSO}_{3}$, sob agitação, até que ocorra a mudança na coloração, do amarelo para verde permanente. Essa mudança de coloração está relacionada à redução do $\mathrm{Cr}(\mathrm{VI})$, que é o estado de oxidação do cromo no cromato, para $\mathrm{Cr}(\mathrm{III})$. Acrescenta-se um pequeno excesso do reagente para que seja garantida a eficácia da reação, pois 0 
bissulfito em $\mathrm{pH}$ neutro tende a formar íon sulfito segundo a reação representada na Eq. 1.

$\mathrm{HSO}_{3}{ }^{-}(\mathrm{aq}) \rightleftharpoons \mathrm{SO}_{3}{ }^{2-}(\mathrm{aq})+\mathrm{H}^{+}(\mathrm{aq})$

Logo em seguida adicionaram-se gotas de $\mathrm{NaOH}$ $6 \mathrm{molL}^{-1}$, sob agitação, até $\mathrm{pH} \geq 10$ para a formação do $\mathrm{Cr}(\mathrm{OH})_{3}$ que é muito pouco solúvel em água; estas duas etapas podem ser representadas pela Eq. 2 e Eq. 3.

$2 \mathrm{CrO}_{4}{ }^{2-}(\mathrm{aq})+3 \mathrm{HSO}_{3}{ }^{-}(\mathrm{aq})+5 \mathrm{H}_{2} \mathrm{O}(\mathrm{l}) \rightarrow 2 \mathrm{Cr}^{3+}(\mathrm{aq})+$

$+3 \mathrm{HSO}_{4}^{-}(\mathrm{aq})+10 \mathrm{OH}^{-}(\mathrm{aq})$

$\mathrm{Cr}^{3+}(\mathrm{aq})+3 \mathrm{OH}^{-}(\mathrm{aq}) \rightarrow \mathrm{Cr}(\mathrm{OH})_{3}(\mathrm{~s})$

O sistema é deixado em repouso pro $24 \mathrm{~h}$, quando é feita novamente uma decantação e filtração para separar o precipitado de $\mathrm{Cr}(\mathrm{OH})_{3}$ do sobrenadante, que deve estar o mais isento possível de cromo para que, então, possa ser descartado adequadamente de acordo com a resolução №357/2005 CONAMA, a saber: o sobrenadante deve ser descartado na pia sob a água corrente, se a concentração de cromo total for igual ou menor que $0,5 \mathrm{mgL}^{-1}$; se estiver acima é necessário realizar o tratamento novamente até que o sobrenadante esteja apto a ser descartado. O precipitado de $\mathrm{Cr}(\mathrm{OH})_{3}$ é seco em estufa a $110^{\circ} \mathrm{C}$ para armazenamento correto.

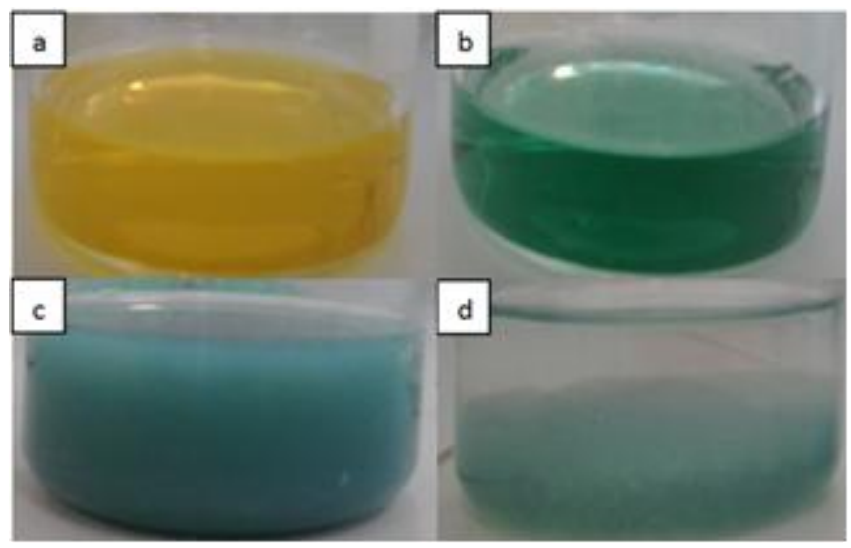

Figura 4 - (a) Sobrenadante filtrado, (b) Adição do agente redutor, (c) Adição de $\mathrm{NaOH}$, (d) Decantação

Fonte: Elaborado pelos autores

\subsection{EVAPORAÇÃO DE ÁGUA, TEMPERATURA E CUSTOS}

Foi feito um estudo com relação à taxa de evaporação do resíduo filtrado no evaporador solar. Para isso, o material foi disposto em diferentes béqueres, fazendo um total de $8900 \mathrm{~mL}$. Durante 22 dias o volume do resíduo contido em cada béquer foi medido e por diferença (Volume anterior - Volume atual) verificouse a quantidade de água evaporada.

A temperatura também foi estudada, por um período de 25 dias (30 de Setembro e 25 de Outubro de 2013). Para medir esse parâmetro utilizou-se, de forma continua durante o período do experimento, um termômetro a álcool que ficava em contato com o piso do evaporador.

Para verificar a quantidade de energia elétrica gasta na pré-concentração utilizando chapa aquecedora (marca QUIMIS, modelo 261,2 e potência de 350 Watts) seguiu-se o procedimento: $\mathrm{Em}$ um béquer foram colocados $200 \mathrm{~mL}$ do resíduo filtrado e este foi aquecido na chapa a temperatura máxima $\left(400^{\circ} \mathrm{C}\right)$ e com velocidade de agitação de $150 \mathrm{rpm}$. No decorrer de $1 \mathrm{~h}$ o volume evaporado foi medido, sendo este parâmetro utilizado para valorar o custo de energia elétrica suficiente para a etapa de pré-concentração.

O fluxograma (FIG. 5) apresenta a sequência dos procedimentos para a realização da gestão do resíduo desde a coleta até a disposição final. 


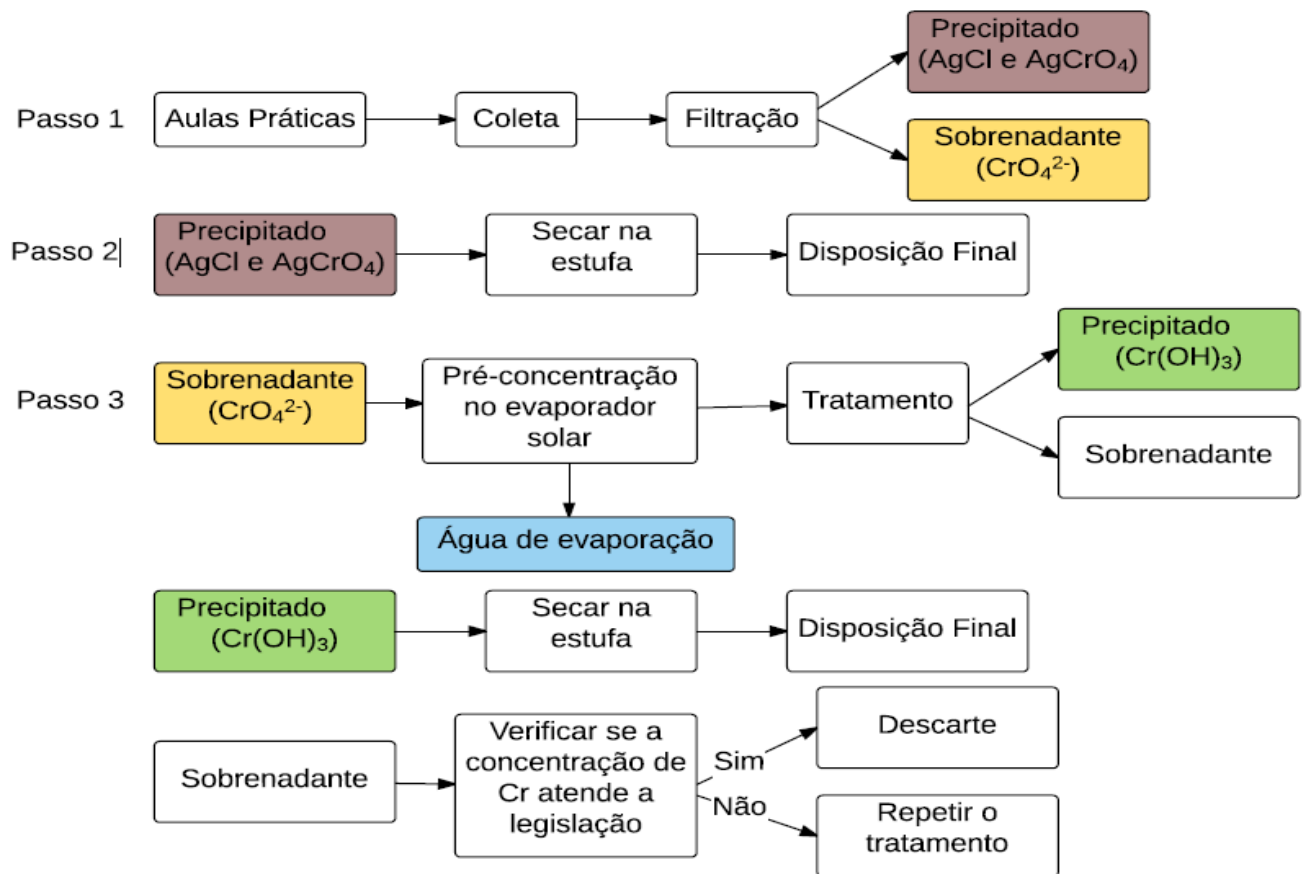

Figura 5 - Fluxograma da gestão do resíduo do método de Mohr.

\section{Resultados e discussão}

\subsection{CONCENTRAÇÃO}

O tratamento do resíduo é mais eficiente, quando na pré-concentração o filtrado adquire uma coloração amarelo escura. Quando se aumenta a concentração de cromo, por adição de mais filtrado ao recipiente em processo de pré-concentração, verifica-se a intensificação da coloração, dificultando a avaliação de efetividade do tratamento posterior do resíduo.

A FIG. 6 exemplifica as diferenças visuais entre 0 sobrenadante que está apto para o procedimento e o que não está apto para o mesmo.

No caso do resíduo não apto, após o tratamento, com formação de precipitado, o sobrenadante ainda apresentou a coloração amarela, indicativo da presença de $\mathrm{Cr}(\mathrm{VI})$, então foi necessária a repetição do tratamento até que sobrenadante não apresentasse visualmente a coloração amarela.

\begin{tabular}{|c|c|c|c|}
\hline Tratamento & Residuo & Tratamento & Decantaçáo \\
\hline Apto & & & \\
\hline & & & \\
\hline
\end{tabular}

Figura 6 - Diferenças entre o resíduo apto e não apto para tratamento

\subsection{EVAPORAÇÃo DE ÁGUA, TEMPERATURA E CUSTOS}

A taxa média de evaporação diária do evaporador solar no período avaliado (22 dias) foi de $501,09 \mathrm{mLdia}^{-1}$ com desvio padrão de 191,38 $\mathrm{mLdia}^{-1}$.

No período do estudo da temperatura (25 dias) a média da temperatura foi $57,69^{\circ} \mathrm{C}$ com desvio padrão de $8,97{ }^{\circ} \mathrm{C}$, sendo que a temperatura mais alta alcançada foi $74^{\circ} \mathrm{C}$. 
No dimensionamento do custo de energia elétrica utilizando a chapa aquecedora, verificou-se que no intervalo de $1 \mathrm{~h}$ foram eliminados $50 \mathrm{~mL}$ do resíduo filtrado, com o custo de $\mathrm{R} \$ 0,33$, considerando a tarifa do consumo faturado de ponta para a Universidade Federal do Ceará, que é de $\mathrm{R} \$ 0,93631 / 1 \quad \mathrm{KWh}$ (Abril/2014). Para a realização do estudo ora apresentado foram utilizados $65 \mathrm{~L}$ de resíduo do método de Mohr. Então, para que esse volume fosse reduzido à metade $\quad(32,5 \mathrm{~L})$, seriam necessárias $650 \mathrm{~h}$ com o custo de $\mathrm{R} \$ 213,01$ para a perda de líquido utilizando a chapa aquecedora, sem considerar a energia gasta pelo exaustor. Considera-se ainda que o custo para a compra de uma chapa aquecedora do modelo utilizado no trabalho é de $\mathrm{R} \$ 999,86$ (sem frete), sendo mais elevado do que o custo para a construção do evaporador solar que é aproximadamente $\mathrm{R} \$ 350$.

Assim, com a utilização do evaporador solar neste trabalho houve uma economia de aproximadamente $\mathrm{R} \$ 862,87$ no processo de pré-concentração de $65 \mathrm{~L}$ do material residual.

\subsection{QUALIDADE DA ÁGUA DE EVAPORAÇÃo}

O sobrenadante do resíduo filtrado (SRF), o sobrenadante recuperado do tratamento com bissulfito de sódio (STB) e a água de evaporação ( $A E$ ) foram submetidos aos testes qualitativos, recomendados pela literatura (VOGEL, 1981), análise por absorção atômica e verificação de condutividade.

\subsubsection{Testes Qualitativos}

Os testes qualitativos foram negativos para todos os íons analisados na água de evaporação, enquanto para o sobrenadante tratado com bissulfito de sódio apesar de não apontar presença do íon cromato, os íons $\mathrm{SO}_{4}{ }^{2-}, \mathrm{SO}_{3}{ }^{2-}$ e $\mathrm{S}_{2} \mathrm{O}_{3}{ }^{2-}$ aparecem devido aos processos do tratamento, como apresenta o FIG.7.

\begin{tabular}{|c|c|c|c|}
\hline & $\mathrm{SRF}$ & $\mathrm{STB}$ & $\mathrm{AE}$ \\
\hline $\mathrm{CrO}_{4}{ }^{2-}$ & $\checkmark$ & - & - \\
\hline $\mathrm{Ca}^{2+}$ & - & - & - \\
\hline $\mathrm{CO}_{3}{ }^{2-}$ & $\checkmark$ & $\checkmark$ & - \\
\hline $\mathrm{Ag}^{+}$ & - & - & - \\
\hline $\mathrm{Cl}^{-}$ & - & - & - \\
\hline $\mathrm{SO}_{4}{ }^{2-}$ & - & $\checkmark$ & - \\
\hline $\mathrm{SO}_{3}{ }^{2-}$ & - & $\checkmark$ & - \\
\hline $\mathrm{S}_{2} \mathrm{O}_{3}{ }^{2-}$ & - & - & - \\
\hline
\end{tabular}

$(-)$ : ausente $(\checkmark)$ : presente

Figura 7 - Resultado dos testes qualitativos da água de evaporação.

\subsubsection{ABSORÇÃO ATÔMICA}

A análise por absorção atômica mostra que a concentração de cromo total na água de evaporação é 47\% menor do que o limite da resolução N³57/2005 CONAMA que é de $0,5 \mathrm{mgL}^{-1}$, enquanto 0 sobrenadante tratado com bissulfito de sódio está $9 \%$ maior. A TAB. 1 exibe os resultados da análise.

Tabela 1

Resultado da análise por absorção atômica da água de evaporação

\begin{tabular}{c|c}
\hline Amostra & $\mathrm{Cr}\left(\mathrm{mgL}^{-1}\right)$ \\
\hline SRF & 263 \\
STB & 0,545 \\
AE & 0,235 \\
\hline
\end{tabular}

A água de evaporação apresentou uma redução da concentração de cromo total no efluente em $99,91 \%$ e o sobrenadante tratado com bissulfito de sódio foi $99,79 \%$. Assim, tanto a água de evaporação quanto o sobrenadante tratado com bissulfito de sódio podem ser descartadas adequadamente. 


\subsubsection{CONDUTIVIDADE}

A condutividade foi verificada utilizando um condutivímetro da marca Micronal e modelo B330, o resultado está exposto na TAB. 2.

Tabela 2

Condutividade

\begin{tabular}{c|c}
\hline Amostra & Condutividade específica $\left(\mathrm{Scm}^{-1}\right)$ \\
\hline AD & $2,365 \times 10^{-5}$ \\
ASA & $9,320 \times 10^{-2}$ \\
AE & $3,423 \times 10^{-2}$ \\
STB & $2,817 \times 10^{-1}$
\end{tabular}

*Água destilada utilizada nos laboratórios de ensino do Departamento de Química Analítica e Físico-Química **Água do sistema de abastecimento do Ceará fornecido pela Companhia de Água e Esgoto do Ceará (CAGECE).

A água de evaporação apresenta condutividade inferior à água do sistema de abastecimento e do sobrenadante tratado com bissulfito de sódio, mas é maior do que da água destilada.

\subsection{DESCARTE DA ÁGUA DE EVAPORAÇÃo}

Pode-se observar por meio dos dados obtidos (TAB. 1) que tanto a água de evaporação quanto 0 sobrenadante tratado podem ser descartados na pia com ou sem diluição. Entretanto, a resolução N³57/2005 CONAMA, diz que o efluente só poderá ser lançado, direta ou indiretamente, nos corpos de água, se o seu $\mathrm{pH}$ estiver ente 5 e 9 . Como no processo de precipitação do $\mathrm{Cr}(\mathrm{III})$ por meio do referido tratamento é necessário que o meio esteja com $\mathrm{pH} \geq 10$, o sobrenadante tratado com bissulfito de sódio não pode ser descartado sem realização de neutralização. Quanto à água de evaporação, por apresentar o valor de $\mathrm{pH}$ na faixa requerida pode ser descartada sem qualquer tratamento prévio, ou reutilizada em manutenção de jardins, diminuindo a demanda de água tratada para este fim.

\section{CONSIDERAÇõES}

Com a utilização do evaporador solar para a préconcentração do resíduo oriundo do método do Mohr houve a minimização de riscos de acidentes, bem como a economia com energia elétrica em $R \$ 862,87$. O evaporador solar mostrou-se muito eficiente, pois a água resultante da evaporação, devido à baixa concentração de cromo total remanescente $\left(0,235 \mathrm{mgL}^{-1}\right)$ e seu $\mathrm{pH} 7$, pode ser reutilizada, ao invés de ser descartada, para manutenções de jardins, diminuindo a utilização de água tratada para este fim.

É importante ressaltar que o evaporador solar utilizado no presente trabalho não pode ser utilizado em época chuvosa, pois os materiais que o compõem não resistem a tal condição, podendo ocorrer infiltrações, crescimento de fungos e 0 arraste de substâncias solúveis em água presentes na madeira. A construção de um evaporador de metal poderia ter uma melhor eficiência.

\section{REFERÊNCIAS}

CAVALCANTE, D. A.; SILVA, M. S. P., BORGES, S. S. S. Gerenciamento de resíduos químicos nos laboratórios de graduação de química analítica quantitativa da Universidade Federal do Ceará. In: Simpósio Nacional sobre Tratamento de Resíduos de Laboratório, 2, 2012, Rio de Janeiro. Resumos do $2^{\circ}$ Simpósio Nacional sobre Tratamento de Resíduos de Laboratório, 2012.

CONAMA, CONSELHO NACIONAL DO MEIO AMBIENTE. Ministério do Meio Ambiente.

Resolução

№357, de 17 de março de 2005. Brasília, DF. 
GIOLI-LIMA, P. C.; LIMA, V. A. Gestão integrada de resíduos químicos em instituições de ensino superior. Química Nova, São Paulo, v. 31, n. 6, 1595 - 1598, ago. ISSN 0100-4042

GUTTERRES, M. SILVA, M. C.; DETTIMER, A. Dossiê sobre Cromo. 2011.

OLIVEIRA, E. S. et al.. Programa de Gerenciamento de Resíduos da UFC: Inventário geral dos resíduos de laboratórios de ensino, pesquisa e extensão. In:

Congresso Brasileiro de Química, 50, 2010, Cuiabá. Anais. 2010. Disponível em:

<http://www.abq.org.br/cbq/2010/trabalhos/5/5-69-

8041.htm>. Acesso em: 21.jul. 2014.

\section{PAULINO, C.V.H. Tendências de Hidrólise dos}

Compostos de $\mathrm{Cr}$ (III) com Ácidos

Poliaminocarboxílicos. 1993. Dissertação de

Mestrado (Química) - Pontifícia Universidade Católica, Rio de Janeiro, 1993.
PENATTI, F. E.; GUIMARÃES, S. T. L.; SILVA, P. M. Gerenciamento de resíduos químicos em laboratórios de análises e pesquisa: o desenvolvimento do sistema em laboratórios da área química. In: Workshop Internacional em Indicadores de Sustentabilidade, 2, 2008, São Carlos. Anais. 2008. 106 - 119.

Disponível em:

<http://www.fsp.usp.br/siades/documentos/Publicacoe s/artigo_9f.pdf>. Acesso em: 15 abr. 2014.

SILVA, J.M. Gerenciamento de Resíduos Químicos no Laboratório de Química Analítica Quantitativa: uma contribuição para o tratamento do resíduo do Método de Mohr. 2014. 42. Monografia de

Graduação (Química) - Centro de Ciências,

Universidade Federal do Ceará, Fortaleza, 2014.

VOGEL, A. I.; Química Analítica Qualitativa. 5. São Paulo: Mestre Jou,1981. 